УДК 528.8/.37/.38

\title{
ТЕХНОЛОГИЧЕСКИЕ РЕШЕНИЯ В ОБЛАСТИ ОБЕСПЕЧЕНИЯ ГЕОПРОСТРАНСТВЕННОЙ ИНФОРМАЦИИ О МАГИСТРАЛЬНЫХ ТРУБОПРОВОДАХ И ОБЪЕКТАХ ИХ ИНФРАСТРУКТУРЫ
}

\author{
Аврунев Евгений Ильич1, \\ avrynev_ei@ngs.ru
}

\author{
Уставич Георгий Афанасьевич1, \\ ystavich@mail.ru
}

\author{
Грекова Антонина Олеговна1, \\ antoninaop@mail.ru
}

\author{
Никонов Антон Викторович1,2, \\ sibte@bk.ru
}

\author{
Мелкий Вячеслав Анатольевич³, \\ vamelkiy@mail.ru
} Долгополов Даниил Валентинович4,
daniil.dolgopolov@gmail.com

\footnotetext{
1 Сибирский государственный университет геосистем и технологий, Россия, 630108, г. Новосибирск, ул. Плахотного, 10.

2 АО «Сибтехэнерго», Россия, 630032, г. Новосибирск, ул. Планировочная, 18/1.

3 Институт морской геологии и геофизики ДВО РАН, Россия, 693022, г. Южно-Сахалинск, ул. Науки, 1 Б.

4 АО «СпейсИнфо Геоматикс», Россия, 127490, г. Москва, ул. Декабристов, 51.
}

Актуальность исследования обусловлена тем, что в настоящее время в Российской Федерации для целей обслуживания добычи и разработки полезных ископаемых необходимо выполнять съемочные и геодезические работы, по результатам которых формируются массивы геопространственных данных, которые являются основой для проектирования и обустройства нефртяных и газовых месторождений и являются исходной информацией для трехмерного моделирования земной поверхности, геологических структур месторождений и инфраструктуры трубопроводных систем. Кроме того, геопространственные данные необходимы для подготовки документов при постановке на государственный кадастровый учет и оформления прав землепользования. На основании этого в статье поставлена научно-техническая задача: проанализировать возможности применения различных способов обеспечения геопространственной инфрормацией ГИС трубопроводных систем данными для 3D моделирования исследуемых месторождений и трубопроводов. Корпоративные ГИС наполняют данными о состоянии трубопроводного транспорта, используя информацию с космических съемочных систем с высоким и средним пространственным разрешением, а также материалы съемки с беспилотных летательных аппаратов и воздушного лазерного сканирования. Мониторинг трубопроводных систем осуществляют также с использованием данных наземных геодезических съемок. Одним из распространенных видов современного геодезического оборудования являются высокоточные и точные электронные тахеометры, позволяющие получать геопространственную информацию о местности и антропогенных объектах как в плане, так и по высоте. Высотная составляющая определяется в результате выполнения тригонометрического нивелирования. Применение способа тригонометрического нивелирования особенно актуально при работе в пересеченной местности и неблагоприятных условиях, к которым в частности относятся районы Крайнего Севера.

Цель: проанализировать возможности применения различных способов обеспечения геопространственной информацией ГИС трубопроводных систем: космических съемок с различным пространственным разрешением, данных беспилотных летательных аппаратов и воздушного лазерного сканирования, тригонометрического нивелирования III и IV классов с применением высокоточных электронных тахеометров при формировании массивов геопространственных данных систем мониторинга магистральных трубопроводов.

Методы: дешифрирование аэрокосмических изображений, экспериментальные исследования в полевых условиях с материалами космических съемок, данными беспилотных летательных аппаратов и воздушного лазерного сканирования, использование высокоточных нивелиров и электронных тахеометров для тригонометрического нивелирования, геоинформационные технологии.

Результаты. Выполнена оценка качества геопространственной информации для ГИС-мониторинга трубопроводных систем. Работа показала, что данные дистанционного зондирования Земли с различными пространственными разрешениями, беспилотных летательных аппаратов и воздушного лазерного сканирования позволяют сформировать массивы геопространственных данных, необходимых для служб, эксплуатирующих трубопроводные системы, обеспечивающих плановую привязку с достаточной точностью; при создании высотного геодезического обоснования, когда точность необходимо повысить до III и IV классов, вместо трудоемкого геометрического нивелирования иелесообразно дополнять массивы информацией, полученной методом тригонометрического нивелирования с использованием высокоточных электронных тахеометров.

\section{Ключевые слова:}

Геопространственные данные, дешифрирование аэрокосмических изображений, геоинформационные технологии, беспилотные летательные аппараты, воздушное лазерное сканирование, трехмерная модель земной поверхности, тригонометрическое нивелирование, высокоточный электронный тахеометр. 


\section{Введение}

В настоящее время одним из ключевых направлений развития экономики Российской Федерации, особенно ее цифровой составляющей, является создание трехмерных моделей разрабатываемых месторождений и трубопроводных систем. Такая постановка проблемы обусловлена необходимостью пространственного представления мест прокладки и эксплуатации трубопроводов, планирования их перспективного использования, оформления соответствующих правоустанавливающих документов, внесения кадастровой информации в единый государственный реестр недвижимости (ЕГРН). Пространственное 3Dмоделирование позволяет представить разрабатываемые месторождения нефти и газа в виде цифровой модели местности, в которой отражается рельеф поверхности, геологическая структура и объекты инфраструктуры трубопроводного транспорта.

Разработка корпоративных геоинформационных систем (ГИС) для предприятий трубопроводного транспорта вызывает потребность в информации о состоянии магистральных трубопроводов и их геометрических характеристиках. Дешифрирование материалов дистанционного зондирования производственных объектов действующего нефтегазового комплекса позволяет распознавать площадки магистральных трубопроводов (МТ), определять их габаритные размеры и не- которые характеристики оборудования, расположенного в их пределах, а также оформлять результаты в виде графических схем (рис. 1).

Результаты съёмки трубопроводного оборудования, полученные с разрешением 0,5 м/пикс, обладают информативностью, позволяющей при использовании эксплуатационной или проектной документации дешифрировать объекты в соответствии с требованиями к составу топографических планов масштаба 1:2000, и могут быть использованы для инвентаризации объектов линейной части МТ и площадок нефтеперекачивающих станций.

\section{Данные дистанционного зондирования Земли - основа геопространственной информации трубопроводных систем}

Основным источником информации для решения наибольшего числа задач являются аэрокосмические изображения, получаемые в оптическом диапазоне длин волн. В то же время для решения отдельных задач могут быть востребованы: данные космической радиолокационной съёмки (задачи определения динамики поверхности земли, объектов МТ в зонах сложных природно-климатических условий), тепловой съёмки, материалы воздушного лазерного сканирования (ВЛС) (задачи построения ЦМР и последующего анализа их изменения).
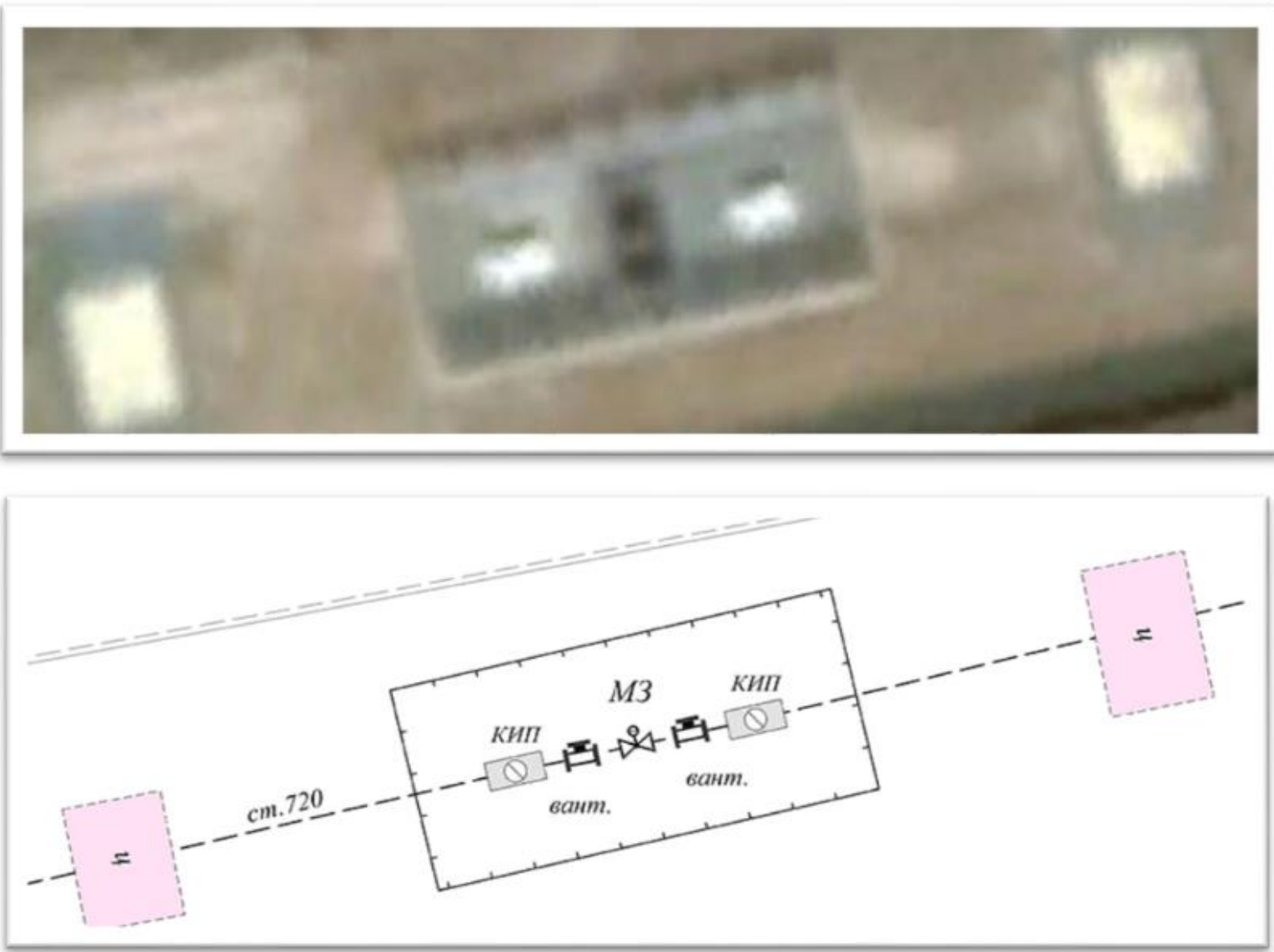

Рис. 1. Пример изображения объекта линейной части трубопровода на космическом снимке (пространственное разрешение 0,5 м/пиксель) (а) и схема, полученная при дешифрировании (б) [1]

Fig. 1. Example of the object on linear part of the pipeline on satellite image (spatial resolution of $0,5 \mathrm{~m} /$ pixel) (a) and schema obtained during decoding (b) [1] 
Для формирования базовой мультимасштабной пространственной основы ГИС могут быть использованы следующие наборы данных дистанционного зондирования Земли (ДЗЗ):

- космическая съемка среднего разрешения (от 5-30 м) обзорное покрытие космической съемкой, данные должны быть представлены на всю территорию России;

- космическая съемка высокого разрешения (1-5 м) базовое покрытие космической съемкой линейной части и прилегающих территорий объектов инфраструктуры МТ (не менее 10 км от оси МТ);

- космическая съемка сверхвысокого разрешения $(<1 \mathrm{M})$ используется для детализации базовой пространственной основы вдоль трассы трубопровода.

Для уточнения/детализации базовой картографической основы вдоль коридора трассы могут использоваться данные космической съемки сверхвысокого разрешения $(<1$ м) наряду с другими методами инструментального дистанционного обследования (АФС, ВЛС и т. д.) (табл. 1).

Таблица 1. Пространственное разрешение съемочных систем [1, 2]

Table 1. Spatial resolution of imaging systems [1, 2]

\begin{tabular}{|c|c|c|}
\hline $\begin{array}{c}\text { Тип аэрокосмической } \\
\text { съемки } \\
\text { Type of aerospace survey }\end{array}$ & $\begin{array}{c}\text { Пространств. } \\
\text { разрешение, м/пикс } \\
\text { Spatial resolution, } \\
\text { m/pixels }\end{array}$ & $\begin{array}{c}\text { Съемочная } \\
\text { система } \\
\text { Survey } \\
\text { system }\end{array}$ \\
\hline $\begin{array}{l}\text { Аэрофотосъёмка с БПЛА } \\
\text { ZALA } \\
\text { Aerial photography from } \\
\text { UAV ZALA }\end{array}$ & $0,05-0,08$ & $\begin{array}{c}\text { MSC } \\
\text { Z-16AGRO1 }\end{array}$ \\
\hline $\begin{array}{l}\text { Космическая съемка (КС) } \\
\text { сверхвысокого разрешения } \\
(0,3-1 \text { м/пикс) } \\
\text { Ultra-high-resolution Space } \\
\text { survey (SS) }(0,3-1 \mathrm{~m} / \text { pix) }\end{array}$ & 0,31 & WorldView 3,4 \\
\hline \multirow{2}{*}{$\begin{array}{l}\text { КС высокого разрешения } \\
\text { (1-5 м/пикс) } \\
\text { High-resolution SS } \\
\text { (1-5 m/pix) }\end{array}$} & 2,5 & SPOT 5 \\
\hline & 3,5 & PlanetScope \\
\hline \multirow{2}{*}{$\begin{array}{l}\text { КС среднего разрешения } \\
\text { (5-30 м/пикс) } \\
\text { Medium-resolution SS } \\
\text { (5-30 m/pix) }\end{array}$} & 10,0 & $\begin{array}{c}\text { Sentinel-2A, } \\
2 \mathrm{~B}\end{array}$ \\
\hline & 15,0 & Landsat 8 \\
\hline $\begin{array}{l}\text { КС низкого разрешения } \\
\text { (>30 м/пикс) } \\
\text { Low-resolution SS } \\
\text { (>30 m/pix) }\end{array}$ & 250,0 & Terra (Modis) \\
\hline
\end{tabular}

\section{Обеспечение высокоточной высотной привязки} геопространственных данных

Материалы космической съемки и эксплуатационная документация не всегда являются достаточными источниками для полного отображения на графических схемах объектов и/или их характеристик. Например, сведения о подземных коммуникациях, типе растительности, назначении зданий могут быть получены в результате полевого дешифрирования и полевой досъемки [1].

Для обеспечения высокоточного пространственного моделирования необходимо создавать цифровое обоснование в виде трехмерной координатной системы $(x, y, H)$. До недавнего времени высотная состав- ляющая определялась геометрическим нивелированием с использованием оптических и цифровых нивелиров. Для определения третьей координаты $H$ в этой системе, согласно действующей инструкции по государственному нивелированию I, II, III и IV классов, используется исключительно метод геометрического нивелирования. Этот метод основан на измерении превышения между двумя точками местности с использованием визирной оси нивелира, которая устанавливается строго горизонтально. Поэтому в условиях всхолмленной и заболоченной местности (последняя, в том числе, имеет место в условиях Крайнего Севера) выполнение геометрического нивелирования связано с рядом сложных технологических особенностей: строгое соблюдение равенства плеч при установке нивелира, минимальная высота визирного луча над подстилающей поверхностью и, как следствие этого, существенное увеличение трудоемкости выполнения данного технологического процесса.

В связи с широким внедрением в геодезическое производство высокоточных электронных тахеометров появилась возможность замены трудоемкого геометрического нивелирования на высокотехнологичное тригонометрическое нивелирование (ТН). В этом случае превышение между точками вычисляется по измеренному углу наклона и расстоянию до визирной цели. Такой способ определения превышений исключает отмеченные выше сложности, что существенно повышает производительность труда [3-9].

При проведении геодезических работ широко применяются высокоточные электронные тахеометры, позволяющие измерять углы наклона со средней квадратической ошибкой (СКО) $0,5^{\prime \prime}-2,0^{\prime \prime}$ и расстояния с ошибкой 1,5-2,0 мм [10-14]. Это открывает новые возможности для применения тригонометрического нивелирования и позволяет обеспечить точность получаемых результатов, соответствующих геометрическому нивелированию III и IV классов. Достижение такой точности позволяет решить все отмеченные выше технологические задачи для создания 3D-моделей всех объектов недвижимости, расположенных, в том числе, в районе залежей полезных ископаемых.

При создании высотного обоснования выбор класса нивелирования предлагается осуществлять на основании категории земель, на которых располагается соответствующий объект. Например, на землях населенных пунктов, где отмечается высокая кадастровая стоимость объектов недвижимости, для формирования 3D-модели рекомендуется использовать нивелирование III, а на землях других категорий - нивелирование IV класса. То же касается ответственных объектов трубопроводов.

Создание пространственных моделей является важным шагом не только для формирования геопространственной информации ГИС трубопроводных систем, когда в результате формирования таких моделей появляется возможность контроля строительства соответствующих инженерных сооружений (в том числе МТ), но и для решения разнообразных научно-технических и эксплуатационных задач. 
В настоящее время при определении плоских прямоугольных координат $(x, y)$ точек земной поверхности для построения 2D-моделей применяются следующие основные методы геодезических измерений (независимо или в комбинации): тахеометрическая съемка, спутниковые измерения, лазерное сканирование (наземное, воздушное, а для протяженных объектов и мобильное), аэрофотосъемка и применение беспилотных авиационных систем (БАС). При формировании 3D-моделей способ полярных координат, составной частью которого является тригонометрическое нивелирование, следует признать наиболее доступным и точным. Применение лазерного сканирования и БАС является весьма перспективным $[15,16]$.

Необходимо отметить, что становление и применение 4D-моделирования потребует определения координат $x, y, H$ через определенные промежутки времени $t$. Актуальность этого направления обусловлена необходимостью выполнения мониторинга состояния земной поверхности, на которой расположены системы трубопроводного транспорта и объекты их инфраструктуры.

Различают следующие способы тригонометрического нивелирования: одностороннее, двустороннее и из середины (рис. 2). Одностороннее нивелирование применяется для определения высоты объекта (здания или сооружения), а также отметок пикетных точек при производстве тахеометрической съемки. При одностороннем нивелировании ошибки за влияние вертикальной рефракции и кривизны Земли не исключаются, однако при длине визирного луча до 150-200 м этими ошибками, как правило, пренебрегают.

Сущность двустороннего тригонометрического нивелирования (ДТН) заключается в двукратном измерении превышения между точками 1 и 2: сначала в прямом, а затем, после перестановки местами тахеометра и визирной цели, - в обратном направлении (рис. 3, a). Среднее значение двух полученных при этом превышений освобождается от влияния кривизны Земли, а влияние рефракции в большей степени компенсируется $[17,18]$. Чем меньше интервал времени между измерениями в прямом и обратном направлениях, тем надежнее исключается рефракционная ошибка из результатов нивелирования. Рекомендуется, чтобы указанный интервал времени не превышал получаса. Главным преимуществом двустороннего нивелирования является возможность его выполнения одновременно с созданием планового геодезического обоснования, для чего на точках хода дополнительно измеряются горизонтальные углы.

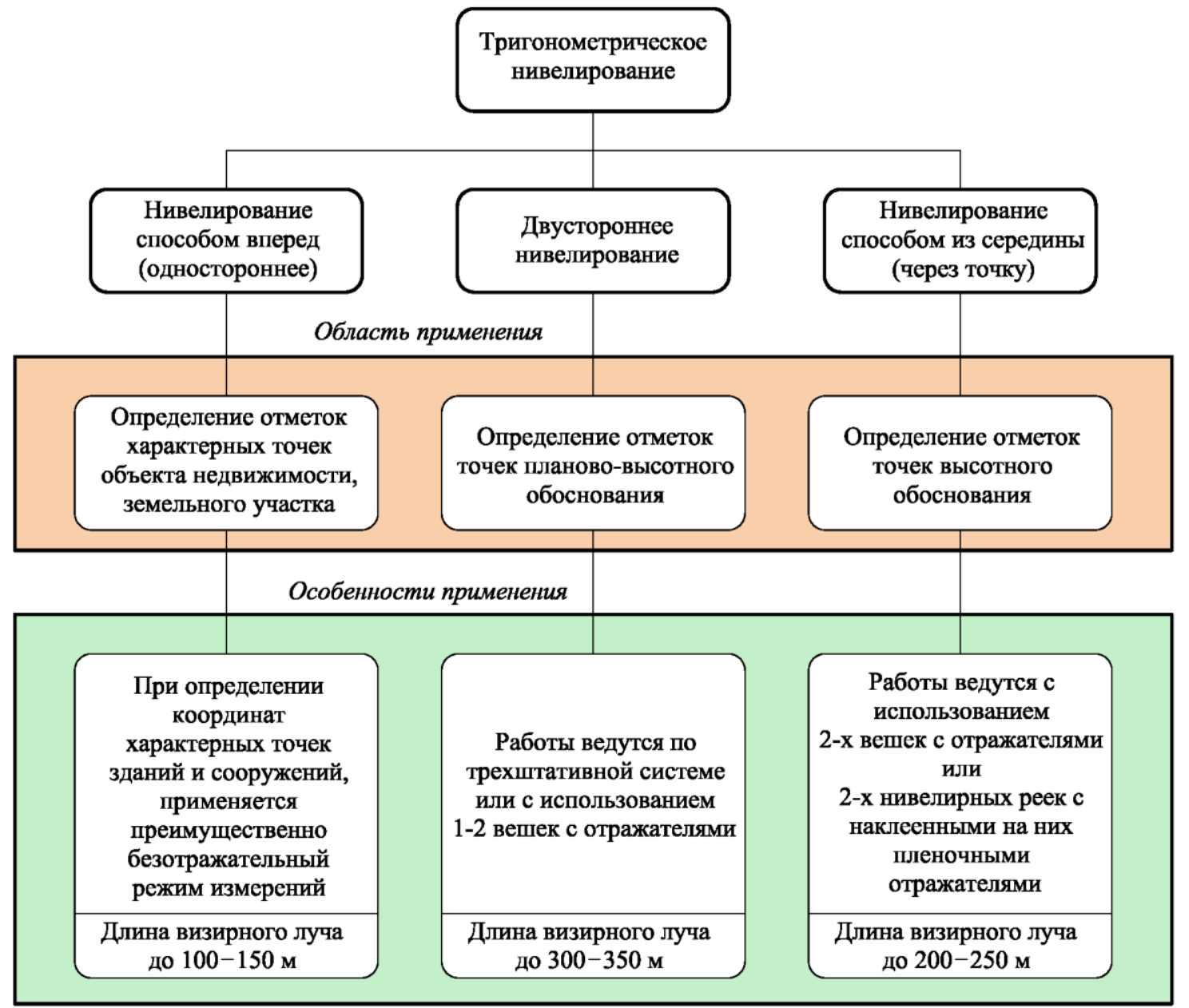

Pис. 2. Способы тригонометрического нивелирования

Fig. 2. Methods of trigonometric leveling 

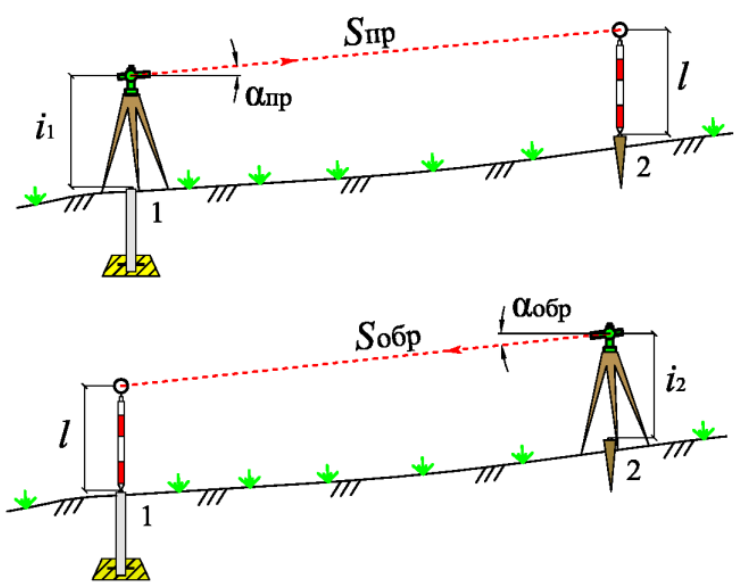

$a / a$
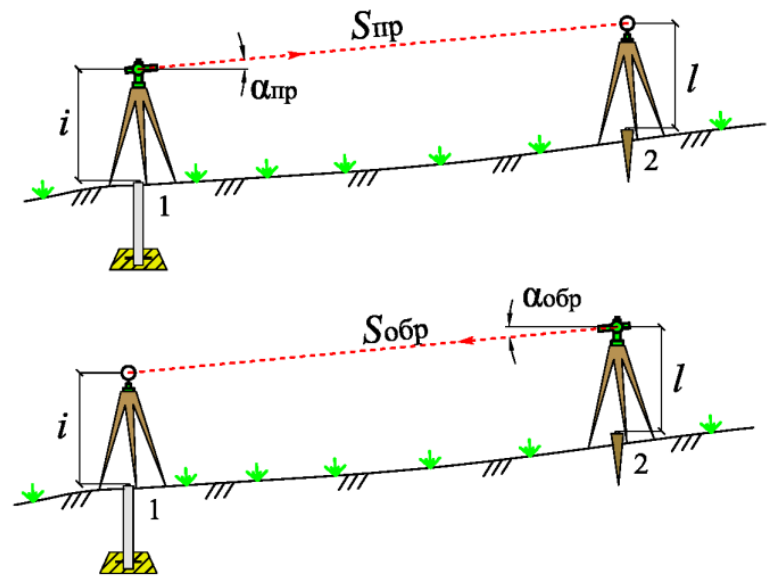

$6 / b$

Рис. 3. Схема выполнения двустороннего нивелирования при использовании вехи с отражателем (а) и при работе по трехитативной системе (б)

Fig. 3. Scheme of reciprocal leveling when using rod with reflector (a) and when working on three-pillar system (b)

Координаты характерных точек объектов повышенной ответственности требуется определять с СКО не более 0,1 м. Съемочное обоснование в этом случае создается методами спутниковых измерений или полигонометрии 4 класса, 1 и 2 разрядов. Выбор метода геодезических построений зависит от удаленности объекта недвижимости или земельного участка от исходных пунктов. При проложении полигонометрических ходов с точностью 1 или 2 разряда (СКО измерения горизонтальных углов $m_{\beta}=5^{\prime \prime}$ и $m_{\beta}=10^{\prime \prime}$ соответственно) в качестве визирной цели применяют призменный отражатель на вехе, которую помощник удерживает на точке руками или с помощью подпорок (рис. 3,a).

Превышение $h_{\text {дв }}$ из двустороннего нивелирования между точкой 1 (репер) и точкой хода 2 , закрепленной на местности колышком (рис. $3, a$ ), находится по формуле

$$
h_{\text {дв }}=\frac{S_{\text {пр }} \sin \alpha_{\text {пр }}-S_{\text {обр }} \sin \alpha_{\text {обр }}}{2}+\frac{i_{1}-i_{2}}{2},
$$

где $S_{\text {пр и }} S_{\text {обр }}$ - наклонные расстояния, измеренные соответственно в прямом и обратном направлениях; $\alpha_{\text {пр и }} \alpha_{\text {обр }}-$ углы наклона; $i_{1}$ и $i_{2}-$ высота инструмента на точках 1 и 2 соответственно.

Как следует из формулы (1), можно не измерять высоту отражателя $l$, если сохранять ее постоянство при измерении превышения в прямом и обратном направлении, чего и следует придерживаться для уменьшения источников ошибок.

Ходы полигонометрии 4 класса (СКО измерения горизонтальных углов $m_{\beta}=2^{\prime \prime}$ ) прокладывают по трехштативной системе. В этом случае отражатели устанавливаются над точками посредством штативов. При перестановке тахеометра с реперной точки 1 на первую точку хода 2 , а отражателя соответственно на точку 1 положение штативов не изменяется: тахеометр и отражатель извлекаются из трегеров и меняются местами. Это позволяет обеспечить неизмен- ность высот $i$ и $l$, а также равенство наклонных расстояний $S_{\text {пр и }} S_{\text {обр }}$ (рис. 3, б). Тем не менее после перестановки тахеометра и отражателя местами для выполнения контроля рекомендуется повторно измерять их высоту над точками 1 и 2. Особенность работы по трехштативной системе заключается в том, что ошибки измерения высот тахеометра и отражателя над точками хода оказывают влияние только на определение отметок собственно переходных точек, но не сказываются на передаче отметки в самом ходе [19].

СКО превышения $m_{h \text { дв }}$ из двустороннего нивелирования, а затем СКО измерения превышения на один километр хода могут быть вычислены по следующим формулам:

$$
m_{h д \mathrm{~B}}=\sqrt{\sin ^{2} \alpha m_{S}^{2}+\frac{S^{2} m_{\alpha}^{2}}{2 \rho^{2}}}, \quad m_{h \text { км }}=m_{h д \mathrm{~B}} \sqrt{n},
$$

где $m_{S}-$ СКО измерения наклонного расстояния; $m_{\alpha}-$ СКО измерения угла наклона одним полным приемом; $\rho=206265^{\prime \prime}$ - число секунд в радиане; $n-$ количество превышений в ходе длиной 1 км.

Результаты предрасчета точности по формуле (2) при $m_{S}=2,0$ мм, $\alpha=6^{\circ}$ (пересеченный рельеф), приводятся в табл. 2 [19].

В инструкции по нивелированию указано, что случайная СКО для нивелирования III класса не должна превышать 5 мм/км, для IV класса -10 мм/км. Из анализа полученных результатов следует, что нормативная точность III класса достигается при ДТН при условии измерения углов наклона с ошибкой не более 3 " и длине линии визирования до 200 м. Точность IV класса соответственно достигается при использовании электронных тахеометров с инструментальной СКО измерения вертикального угла 5" и длине линии визирования до $300 \mathrm{~m}$.

Данные рассуждения справедливы, если ход прокладывается по трехштативной системе, так как лишь в этом случае ошибки измерения высоты тахеометра 
(отражателя) не будут накапливаться. Ошибками измерения высоты инструмента или отражателя над реперами при этом можно пренебречь.

Таблица 2. Средние квадратические ошибки ДТН при различной длине линий визирования

Table 2. $\quad$ Standard deviations of reciprocal trigonometric leveling at different lengths of observing line

\begin{tabular}{|c|c|c|c|c|c|c|c|c|}
\hline \multirow[t]{2}{*}{$m_{\alpha}, \mathrm{c}$} & \multicolumn{3}{|c|}{ 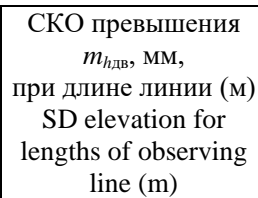 } & \multicolumn{3}{|c|}{\begin{tabular}{|c|} 
СКО превышения на \\
1 км хода, мм, при \\
длине линий (м) \\
SD elevation for $1 \mathrm{~km}$ \\
run for lengths of \\
observing line $(\mathrm{m})$ \\
\end{tabular}} & \multicolumn{2}{|c|}{$\begin{array}{c}\text { Нормативная } \\
\text { СКО на } 1 \text { км } \\
\text { для класса } \\
\text { Normal SD } \\
\text { for } 1 \mathrm{~km} \text { for } \\
\text { class } \\
\end{array}$} \\
\hline & 100 & 200 & 300 & 100 & 200 & 300 & III & IV \\
\hline $1^{\prime \prime}$ & 0,4 & & 1,1 & 1,3 & & & \multirow{5}{*}{$5 \mathrm{MM}$} & \multirow{5}{*}{$10 \mathrm{MM}$} \\
\hline $2^{\prime \prime}$ & 0,7 & & 2,1 & 2,3 & 3,1 & 3,8 & & \\
\hline $3^{\prime \prime}$ & 1,1 & 2,1 & 3,1 & 3,3 & 4,6 & 5,6 & & \\
\hline $5^{\prime \prime}$ & 1,7 & & 5,2 & 5,5 & 7,7 & 9,4 & & \\
\hline $7^{\prime \prime}$ & 2,4 & 4,8 & 7,2 & 7,6 & 10,7 & 13,1 & & \\
\hline
\end{tabular}

Произведем оценку влияния ошибки измерения высоты инструмента $m_{i}$ на точность двустороннего нивелирования при прокладывании хода с отражателем на вехе. Если измерять высоту инструмента рулеткой с ошибкой $m_{i}=2$ мм, то СКО второго слагаемого в формуле (1) также составит 2 мм. Тогда ошибка превышения между точками хода $m_{h д в}^{\prime}$ с учетом $m_{i}$ может быть вычислена формуле

$$
m_{h д \text { дв }}^{\prime}=\sqrt{m_{\text {ддв }}^{2}+m_{i}^{2}} .
$$

Результаты предрасчета точности двустороннего нивелирования с учетом ошибки измерения высоты тахеометра, полученные по формуле (3), приведены в табл. 3.

Из анализа данных табл. 3 следует, что при измерении высоты тахеометра с ошибкой 2 мм и использовании отражателя на вехе точность нивелирования III класса может быть достигнута лишь при значениях ошибок измерения углов, не превышающих 1", чего добиться при воздействии факторов внешней среды весьма затруднительно. В то же время точность IV

класса уверенно обеспечивается при измерении углов с ошибкой $2 "-3$ ".

Рассмотрим третий способ тригонометрического нивелирования - из середины. Тахеометр при этом устанавливается между двумя точками (рис. 4).

При нивелировании способом из середины, как и в двустороннем нивелировании, происходит компенсация влияния кривизны Земли и рефракции [20-22]. Важным достоинством нивелирования из середины является отсутствие необходимости измерять высоту инструмента. Также данный способ является более производительным: при расстоянии от тахеометра до отражателя 200 м обеспечивается передача отметки сразу на 400 м, в то время как в двустороннем нивелировании пришлось бы разбивать это расстояние на две части (точное визирование сразу на 400 м затруднительно).

Формула вычисления превышения при нивелировании из середины при равенстве высот задней и передней визирных целей над точками хода имеет вид

$$
h_{\text {cep }}=S_{2} \sin \alpha_{2}-S_{1} \sin \alpha_{1}-\frac{S^{2}}{2 R}\left(k_{2}-k_{1}\right),
$$

где $k_{1}$ и $k_{2}-$ коэффициент рефракции, соответственно, при визировании на точку 1 и $2 ; R$ - средний радиус Земли (6371 км).

Таблица 3. Средние квадратические ошибки ДТН с учетом ошибки за измерение высоты инструмента

Table 3. Standard deviations of reciprocal trigonometric leveling taking into account errors of horizon of instrument

\begin{tabular}{|c|c|c|c|c|c|c|}
\hline$m_{\alpha}, \mathrm{c}$ & $\begin{array}{c}\text { СКО превышения } m_{\text {hдв }}, \\
\text { мм, при длине линии (м) } \\
\begin{array}{c}\text { SD elevation for sight line } \\
\text { length (m) }\end{array}\end{array}$ & $\begin{array}{c}\text { СКО превышения } m_{\text {нкм на 1 км }} \\
\text { хода, мм, при длине линий } \\
\text { SD elevation for 1 km run for } \\
\text { sight line (m) }\end{array}$ \\
\cline { 2 - 7 } & 100 & 200 & 300 & 100 & 200 & 300 \\
\hline $1^{\prime \prime}$ & 2,0 & 2,1 & 2,3 & 6,4 & 4,8 & 4,1 \\
\hline $2^{\prime \prime}$ & 2,1 & 2,4 & 2,9 & 6,7 & 5,4 & 5,2 \\
\hline $3^{\prime \prime}$ & 2,3 & 2,9 & 3,7 & 7,1 & 6,4 & 6,7 \\
\hline $5^{\prime \prime}$ & 2,6 & 4,0 & 5,5 & 8,4 & 8,9 & 10,1 \\
\hline $7 "$ & 3,1 & 5,2 & 7,5 & 9,9 & 11,6 & 13,6 \\
\hline
\end{tabular}

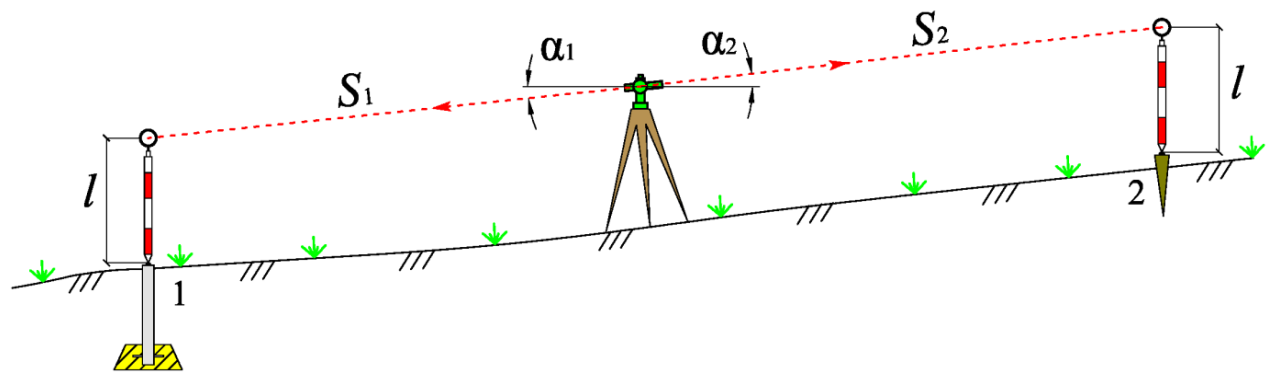

Pис. 4. Схема тригонометрического нивелирования способом из середины

Fig. 4. Scheme of leap-frog trigonometric leveling

Третье слагаемое в формуле (4) обусловлено неравенством коэффициентов рефракции при визировании на точки 1 и 2. В общем случае разность коэффициентов рефракции $\Delta k$ есть величина неизвестная. При однородности подстилающей поверхности выполнение визирования на заднюю и переднюю точки позволяет пренебречь различием коэффициентов рефракции, тогда точность нивелирования можно оценить по формуле

$$
m_{\text {hcep }}=\sqrt{\left(2 \sin ^{2} \alpha\right) m_{S}^{2}+\frac{2 S^{2} m_{\alpha}^{2}}{\rho^{2}}} .
$$


Средние квадратические ошибки измерения превышения при ТН из середины для $\alpha=6^{\circ}$ представлены в табл. 4.

Анализ данных табл. 4 показал, что для выполнения нивелирования с точностью III класса измерения углов наклона следует выполнять с ошибкой не более 2", а длину плеч сократить до $S<200$ м. Тригонометрическое нивелирование с точностью, которую можно отнести к IV классу, следует выполнять тахеометрами со СКО измерения угла наклона менее 3 " и длине плеч не более 200-300 м [20].

Таблица 4. Средние квадратические ошибки ТН способом из серединь

Table 4. Standard deviations of leap-frog trigonometric leveling

\begin{tabular}{|c|c|c|c|c|c|c|}
\hline \multirow[t]{2}{*}{$m_{\alpha}, \mathrm{c}$} & \multicolumn{3}{|c|}{$\begin{array}{c}\text { СКО превышения } m_{h c е p} \\
\text { мм, при длине линии } \\
\text { (м) } \\
\text { SD elevation for sight } \\
\text { line length (m) }\end{array}$} & \multicolumn{3}{|c|}{$\begin{array}{c}\text { СКО превышения } m_{\text {кмм }} \text { на } \\
1 \text { км хода, мм, при длине } \\
\text { линий (м) } \\
\text { SD elevation for } 1 \text { km run for } \\
\text { sight line length (m) }\end{array}$} \\
\hline & 100 & 200 & 300 & 100 & 200 & 300 \\
\hline $1^{\prime \prime}$ & 0,8 & 1,4 & 2,1 & 1,7 & 2,2 & 2,7 \\
\hline $2^{\prime \prime}$ & 1,4 & 2,8 & 4,1 & 3,1 & 4,4 & 5,3 \\
\hline $3^{\prime \prime}$ & 2,1 & 4,1 & 6,2 & 4,7 & 6,5 & 8,0 \\
\hline $5^{\prime \prime}$ & 3,4 & 6,9 & 10,3 & 7,7 & 10,9 & 13,3 \\
\hline $7^{\prime \prime}$ & 4,8 & 9,6 & 14,4 & 10,8 & 15,2 & 18,6 \\
\hline
\end{tabular}

Предварительными вычислениями установлено, что ходы ТН, прокладываемые при использовании высокоточных тахеометров $\left(m_{\alpha} \leq 2^{\prime \prime}\right)$, могут соответствовать по точности геометрическому нивелированию III или IV классов. В целях снижения влияния на результаты нивелирования ошибок, обусловленных вертикальной рефракцией и неустойчивостью штатива или переходных точек, следует рассмотреть некоторые методические особенности при измерениях, выполняемых на станции [18].

Рассмотрим основные требования технологического характера, которые необходимо обеспечивать при выполнении ТН с точностью, предъявляемой к нивелированию III и IV классов:

- порядок взятия отсчетов на станциях (программа измерений);

- допустимая минимальная высота визирования;

- допустимая величина неравенства плеч на станции и допустимое значение его накопления в ходе;

- благоприятное время для выполнения измерений;

- система контролей и допусков на станции.

Предлагаемая методика ТН предусматривает использование высокоточных электронных тахеометров с СКО измерения углов наклона не более 2 ", расстояний с СКО не более 2-3 мм (в том числе в безотражательном режиме) [19].

В качестве визирных целей можно применять нескладные деревянные или металлические нивелирные рейки и вехи с отражателем. При использовании реек измерения расстояний выполняются в безотражательном режиме. Для удобства визирования на рейки рекомендуется наклеивать пленочные отражатели размером $50 \times 50$ мм на расстоянии не менее 1,5 м от пятки рейки. Для удобства обработки результатов измерений при выполнении нивелирования способом из середины высота закрепления пленочных отражателей в паре реек относительно пяток должна быть одинаковой (в пределах 0,2 мм). Для обеспечения равенства высот пленочных отражателей необходимо использовать женевскую линейку.

Если при выполнении нивелирования будут применяться вехи с отражателем, то для установки на переходных точках (костылях) их необходимо снабдить плоской пяткой. Вертикальность вех в процессе измерений обеспечивается с помощью круглых уровней с ценой деления не более 20' (при углах наклона $\alpha \leq 2^{\circ}$ ), а на всхолмленной местности (при углах наклона $\alpha \leq 6^{\circ}$ ) с ценой деления не более $10^{\prime}$. Для установки и фиксации реек или вех в вертикальном положении используют подпорки (биподы).

Для обеспечения контроля измерений, выполненных на станции при расстояниях до рейки свыше 100 м следует определять превышения двумя полными приемами. Для независимости измерений рекомендуется между приемами менять высоту тахеометра подъемными винтами в пределах одного сантиметра. Измерение превышения двумя приемами может выполняться и при одном горизонте тахеометра. В этом случае визирная цель должна представлять собой вертикальный базис, что обеспечит получение второго независимого превышения на станции без изменения высоты тахеометра. За точки вертикального базиса можно принять два сантиметровых деления шашечной рейки или два пленочных отражателя, расстояние между которыми измеряется женевской линейкой с погрешностью не более 0,2 мм (рис. 5, a). Расстояние между точками базиса принимается около 200-500 мм с таким расчетом, чтобы нижний конец базиса имел высоту над пяткой рейки не менее 1,5 м.

Программы измерений на станщии. При выполнении геометрического нивелирования для уменьшения ошибок за выпирание или оседание штатива программу измерений на станции строят таким образом, чтобы все отсчеты по рейкам располагались симметрично относительно некоторого среднего момента времени. По аналогии для ослабления ошибок за перемещение штатива с тахеометром при выполнении тригонометрического нивелирования необходимо также обеспечивать симметричность при наведениях на визирные цели. Кроме того, оптимальной следует считать программу с минимальным количеством переходов наблюдателя вокруг тахеометра.

При выполнении нивелирования III и IV классов следует выполнять измерения по программам, представленным в табл. 5. В данном случае превышение измеряется полными приемами, т. е. при положении вертикального круга слева (КЛ) и справа (КП). При нивелировании с использованием вертикального базиса высота инструмента не изменяется, что позволяет проконтролировать место нуля (МО).

Представленные программы измерений являются симметричными, а наблюдателю требуется всего два раза переходить вокруг прибора. Таким образом, ошибки за перемещение системы «штатив-тахеометр» в полученных превышениях будут несущественны (рис. 6). 

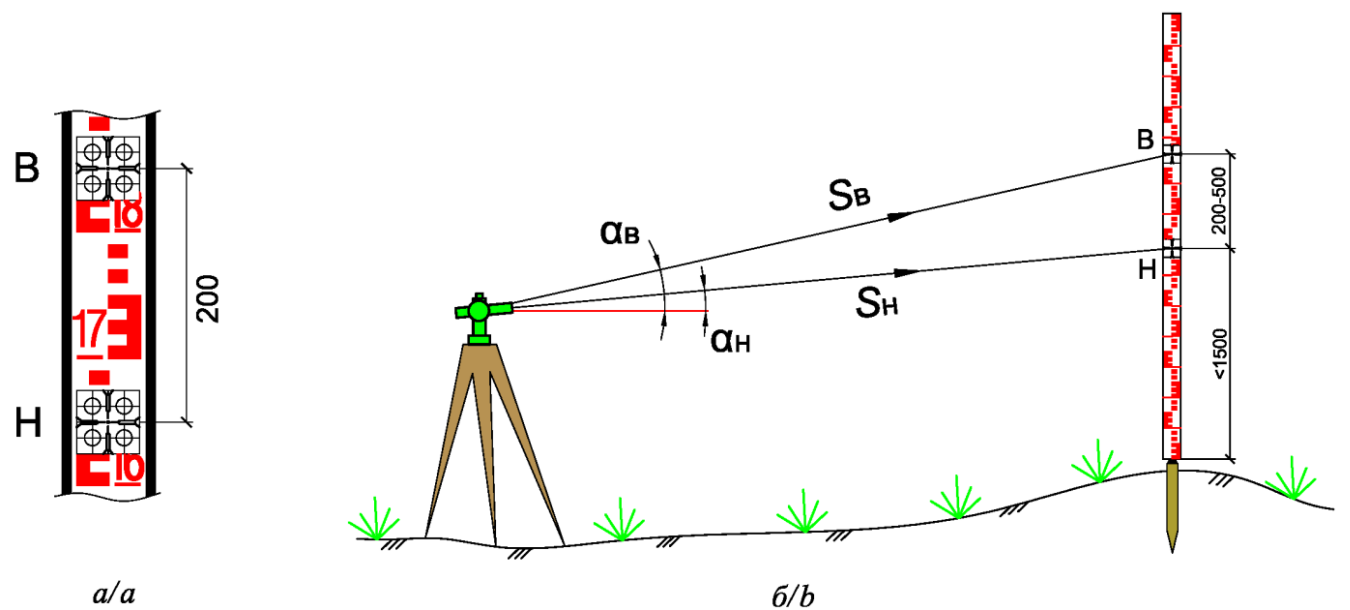

$a / a$

Рис. 5. Фрагмент нивелирной рейки с двумя пленочными отражсателями (а) и схема измерений на вертикальный базис (б)

Fig. 5. Fragment of the leveling rod with two film reflectors (a) and measurement scheme on a vertical basis (b)
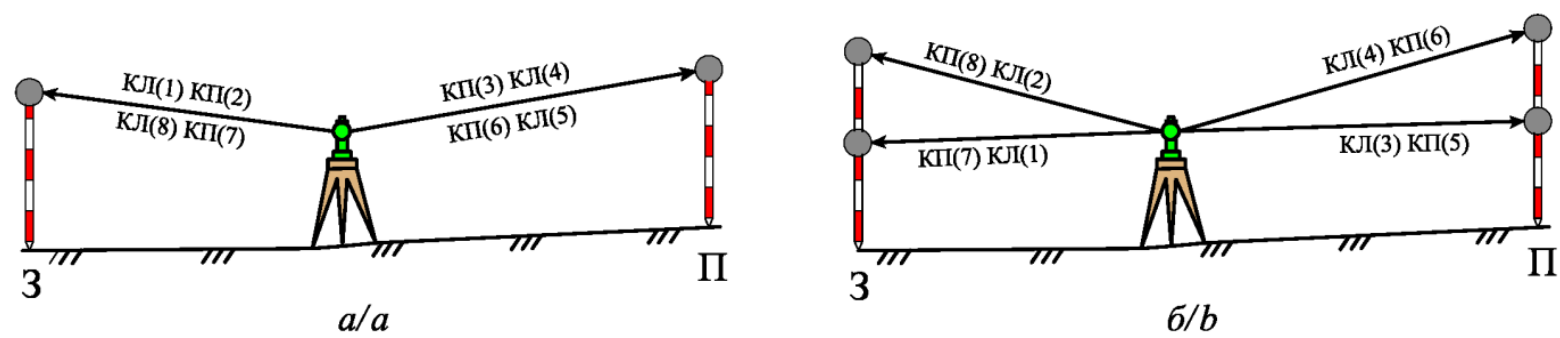

Рис. 6. Программа измерений на станщии при визировании на одинарную цель (а) и вертикальный базис (б)

Fig. 6. Measurement program at the station when sighting on a single target (a) and vertical basis (b)

Для обеспечения стабильного положения системы «штатив-тахеометр» рекомендуется снять дерн под ножками штатива, а измерения начинать спустя 1-2 минуты после установки тахеометра на штатив. Существенно ослабить влияние перемещения переходных точек на результаты нивелирования можно путем использования металлических костылей или кольев длиной $35-40 \mathrm{~cm}$.

Таблица 5. Программы измерений на станции тригонометрического нивелирования

Table 5. Measurement programs on the station of trigonometric leveling

\begin{tabular}{|c|c|c|c|c|c|c|}
\hline \multirow{3}{*}{ 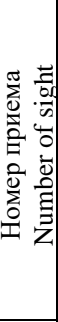 } & \multicolumn{2}{|c|}{$\begin{array}{c}\text { Программа (1) при } \\
\text { визировании } \\
\text { на одинарную цель } \\
\text { (отражатель) } \\
\text { Program (1) in sighting } \\
\text { on a single target (reflector) }\end{array}$} & \multicolumn{4}{|c|}{$\begin{array}{c}\text { Программа (2) при } \\
\text { визировании } \\
\text { на вертикальный базис } \\
\text { Program (2) in sighting } \\
\text { on vertical basis }\end{array}$} \\
\hline & \multirow{2}{*}{$\begin{array}{c}\text { задняя } \\
\text { цель } \\
\text { back target }\end{array}$} & \multirow{2}{*}{$\begin{array}{c}\text { передняя } \\
\text { цель } \\
\text { front target }\end{array}$} & \multicolumn{2}{|c|}{$\begin{array}{c}\text { задняя цель } \\
\text { back target }\end{array}$} & \multicolumn{2}{|c|}{$\begin{array}{c}\text { передняя цель } \\
\text { front target }\end{array}$} \\
\hline & & & $\begin{array}{c}\text { низ } \\
\text { bottom }\end{array}$ & $\begin{array}{c}\text { верх } \\
\text { top }\end{array}$ & $\begin{array}{c}\text { низ } \\
\text { bottom }\end{array}$ & $\begin{array}{c}\text { верx } \\
\text { top }\end{array}$ \\
\hline \multirow{2}{*}{1} & КЛ (1) & КП (3) & \multirow{2}{*}{ КЛ (1) } & \multirow{2}{*}{ КЛ (2) } & \multirow{2}{*}{ КЛ (3) } & \multirow{2}{*}{ КЛ (4) } \\
\hline & КП (2) & КЛ (4) & & & & \\
\hline \multirow{2}{*}{2} & КП (7) & КЛ (5) & \multirow{2}{*}{ КП (7) } & \multirow{2}{*}{ КП (8) } & \multirow{2}{*}{ КП (5) } & \multirow{2}{*}{ КП (6) } \\
\hline & КЛ (8) & КП (6) & & & & \\
\hline
\end{tabular}

Допустимая минимальная высота визирования. Для уменьшения рефракционного влияния на измеренные превышения визирный луч должен проходить на расстоянии не менее 1 м над подстилающей по- верхностью, а высоту визирной цели следует принимать не менее 1,5 м. Для сравнения укажем, что при геометрическом нивелировании III и IV классов, согласно инструкции, высота визирования должна быть не менее 0,3 и 0,2 м соответственно.

Время выполнения измерений. Как и при работе с нивелиром, измерения следует проводить при хорошей видимости, отчетливых и спокойных изображениях визирных целей. В ясную солнечную погоду следует исключить измерения в течение часа после восхода и за час до захода Солнца. При нивелировании в жаркие, солнечные дни необходимо уменьшать расстояния до реек и соблюдать однородность подстилающей поверхности при наведении на заднюю и переднюю визирные цели.

Допустимая величина неравенства плеч на станияи и его накопления в ходе. При выполнении нивелирования расстояния до целей измеряют светодальномерным блоком тахеометра. При нивелировании III и IV классов неравенство плеч на станции не должно превышать 10 и 20 м соответственно, а его накопление по секции - 20 м. В исключительных случаях, когда в силу местных условий не получается установить тахеометр на равных расстояниях от визирных целей, допускается неравенство плеч до 30 м, при условии последующей единовременной его компенсации на одной из последующих станций хода.

Длины визирных лучей при выполнении тригонометрического нивелирования III и IV классов целесообразно ограничить величиной 200 и 250 м соответ- 
ственно (табл. 6), так как при больших расстояниях ошибки рефракционного характера начинают превалировать. Заметим, что для геометрического нивелирования инструкцией допускается предельная длина визирного луча 100 м (III класс) и 150 м (IV класс). Таким образом, применение тахеометров для нивелирования III и IV классов позволяет увеличить расстояние до визирных целей до 2 раз, снизить количество переходных точек и тем самым повысить производительность работ.

Таблица 6. Требования к тригонометрическому нивелированию III и IV классов способом из середи$\mathrm{Hbl}$

Table 6. Requirements for leap-frog trigonometric leveling classes III and IV

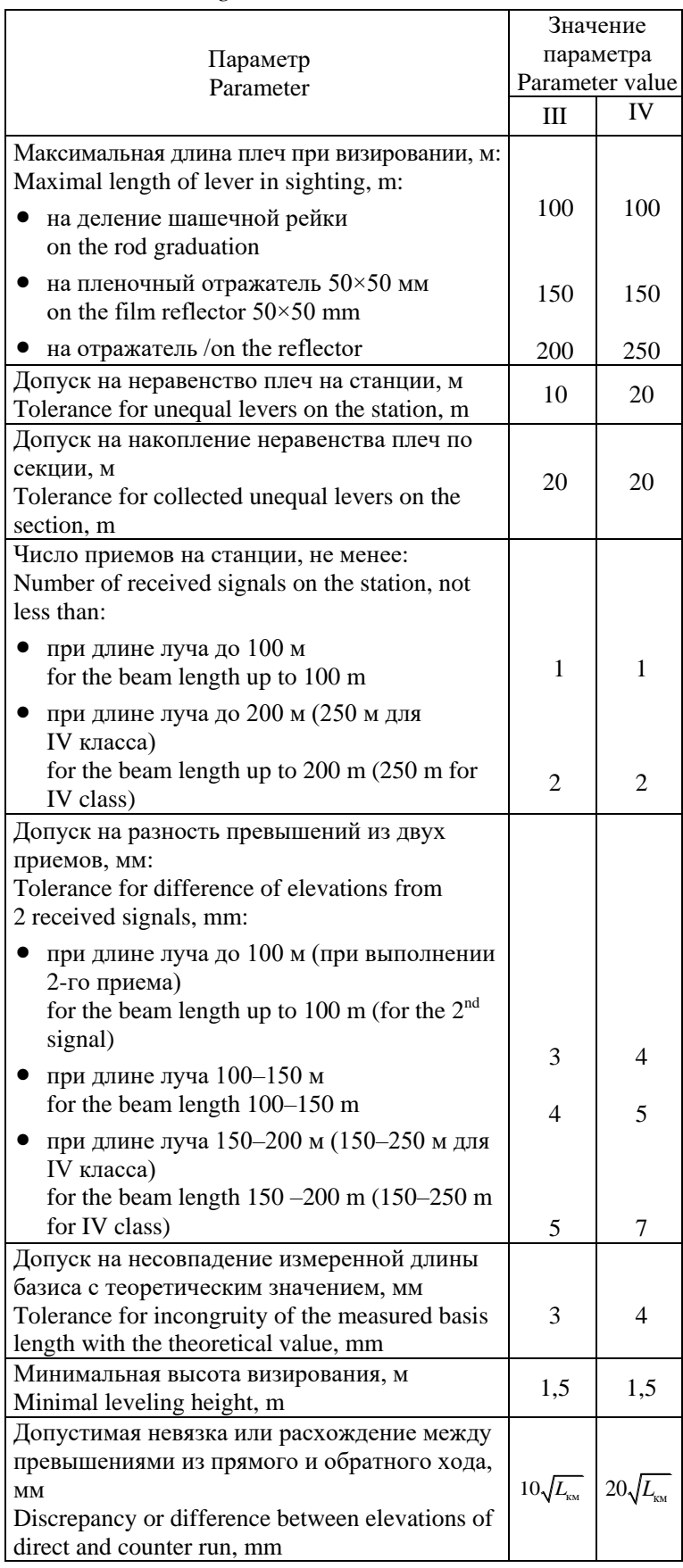

Система контролей и допусков на станщии. При тригонометрическом нивелировании на станции превышение измеряется между осью вращения зрительной трубы тахеометра и центром каждой визирной цели при двух положениях круга (КЛ и КП). Из-за перепадов температуры воздуха МО может изменяться и разность превышений, полученная при КЛ и КП, не будет величиной постоянной для данного расстояния. Поэтому контроль измерений должен заключаться в сравнении между собой превышений между задней и передней точками из двух приемов. Использование вертикального базиса известной длины дает дополнительный контроль качества измерений. В этом случае разность превышений при визировании на нижний и верхний концы базиса должна равняться длине базиса.

При выполнении двустороннего тригонометрического нивелирования следует соблюдать требования, представленные в табл. 7.

Таблица 7. Требования к двустороннему тригонометрическому нивелированию III и IV классов

Table 7. Requirements for reciprocal trigonometric leveling classes III and IV

\begin{tabular}{|c|c|c|}
\hline \multirow[t]{2}{*}{$\begin{array}{l}\text { Параметр } \\
\text { Parameter }\end{array}$} & \multicolumn{2}{|c|}{$\begin{array}{l}\text { Значение пара- } \\
\text { метра для класса } \\
\text { Parameter value } \\
\text { for class }\end{array}$} \\
\hline & III & IV \\
\hline $\begin{array}{l}\text { Паспортная точность измерения угла } \\
\text { наклона, не более, с } \\
\text { Potential accuracy of inclination angle } \\
\text { measurement, not more than, s }\end{array}$ & $2^{\prime \prime}$ & $2^{\prime \prime}$ \\
\hline $\begin{array}{l}\text { Максимальная длина стороны хода, м: } \\
\text { Maximal legnth of run side, m: }\end{array}$ & 300 & 350 \\
\hline $\begin{array}{l}\text { Число приемов на станции, не менее: } \\
\text { Number of received signals on the station, } \\
\text { not less than: } \\
\text { • при длине луча до } 150 \text { м } \\
\text { for the beam length up to } 150 \mathrm{~m} \\
\text { - при длине луча свыше } 150 \mathrm{~m} \\
\text { for the beam length over } 150 \mathrm{~m}\end{array}$ & 1 & $\begin{array}{l}1 \\
2\end{array}$ \\
\hline $\begin{array}{l}\text { Допуск на разность превышений из двух } \\
\text { приемов, мм: } \\
\text { Tolerance for elevation difference from two } \\
\text { signals, mm: } \\
\text { - при длине луча до } 150 \text { м (при выпол- } \\
\text { нении 2-го приема) } \\
\text { for the beam length up to } 150 \mathrm{~m} \text { (for the } \\
\text { 2nd signal) } \\
\text { - } \text { при длине луча от } 150 \text { до } 300 \mathrm{м} \\
\text { for the beam length from } 150 \text { to } 300 \mathrm{~m}\end{array}$ & 4 & $\begin{array}{l}5 \\
8\end{array}$ \\
\hline $\begin{array}{l}\text { Минимальная высота визирования, м } \\
\text { Minimal sighting height, m }\end{array}$ & 1,5 & 1,5 \\
\hline $\begin{array}{l}\text { Допустимая невязка или расхождение } \\
\text { между превышениями из прямого и об- } \\
\text { ратного хода, мм } \\
\text { Discrepancy or difference between } \\
\text { elevations of direct and counter run, mm }\end{array}$ & $10 \sqrt{L_{\mathrm{kM}}}$ & $20 \sqrt{L_{\text {км }}}$ \\
\hline
\end{tabular}

В качестве визирных целей следует использовать круглые отражатели. Двустороннее тригонометрическое нивелирование III класса допускается выполнять только по трехштативной системе. Допуски на разность превышений, полученных из измерений между 
двумя точками в прямом и обратном направлениях, нами не устанавливались. Это обусловлено тем, что указанные разности определяются не только точностью собственно измерений, но и в значительной степени влиянием вертикальной рефракции. Например, в статье [20] указывается, что для расстояния $400 \mathrm{~m}$ разность превышений из измерений в прямом и обратном направлениях из-за систематического влияния рефракции может достигать 80 мм.

\section{Оценка точности превышений}

\section{при тригонометрическом нивелировании}

Для проверки теоретических положений и правильности выполненного предрасчета точности тригонометрического нивелирования летом 2018 г. были проведены полевые исследования. Работы выполнялись при температуре воздуха $+23{ }^{\circ} \mathrm{C}$ и переменной облачности. На местности были выбраны два грунтовых репера $R p 1$ и $R p 2$, удаленных друг от друга на расстояние 1,1 км. Между реперами примерно через
100 м было забито в грунт 10 деревянных кольев длиной $0,3 \mathrm{~m}$.

Предварительно, по закрепленным точками T.1-Т.10, по программе II класса был проложен ход геометрического нивелирования (рис. 7, а). Подстилающая поверхность представляла собой невысокую травянистую растительность, что в значительной степени ослабило влияние вертикальной рефракции. Работы выполнялись цифровым нивелиром DiNi 0.3 в комплекте с двумя штрих-кодовыми рейками с инварной полосой длиной $2 \mathrm{M}$. Паспортная точность нивелира составляет 0,3 мм/км двойного хода. Ход прокладывался в двух направлениях, расстояние от нивелира до рейки составляло примерно 50 м. Разность превышений из прямого и обратного хода составила 1,9 мм, что не превышает допустимого значения $f_{\text {доп }}=5 \mathrm{~mm} \sqrt{1,1 \text { км }}=5,2$ мм. За окончательные (эталонные) превышения между реперами и кольями были приняты средние значения превышений из ходов в двух направлениях.
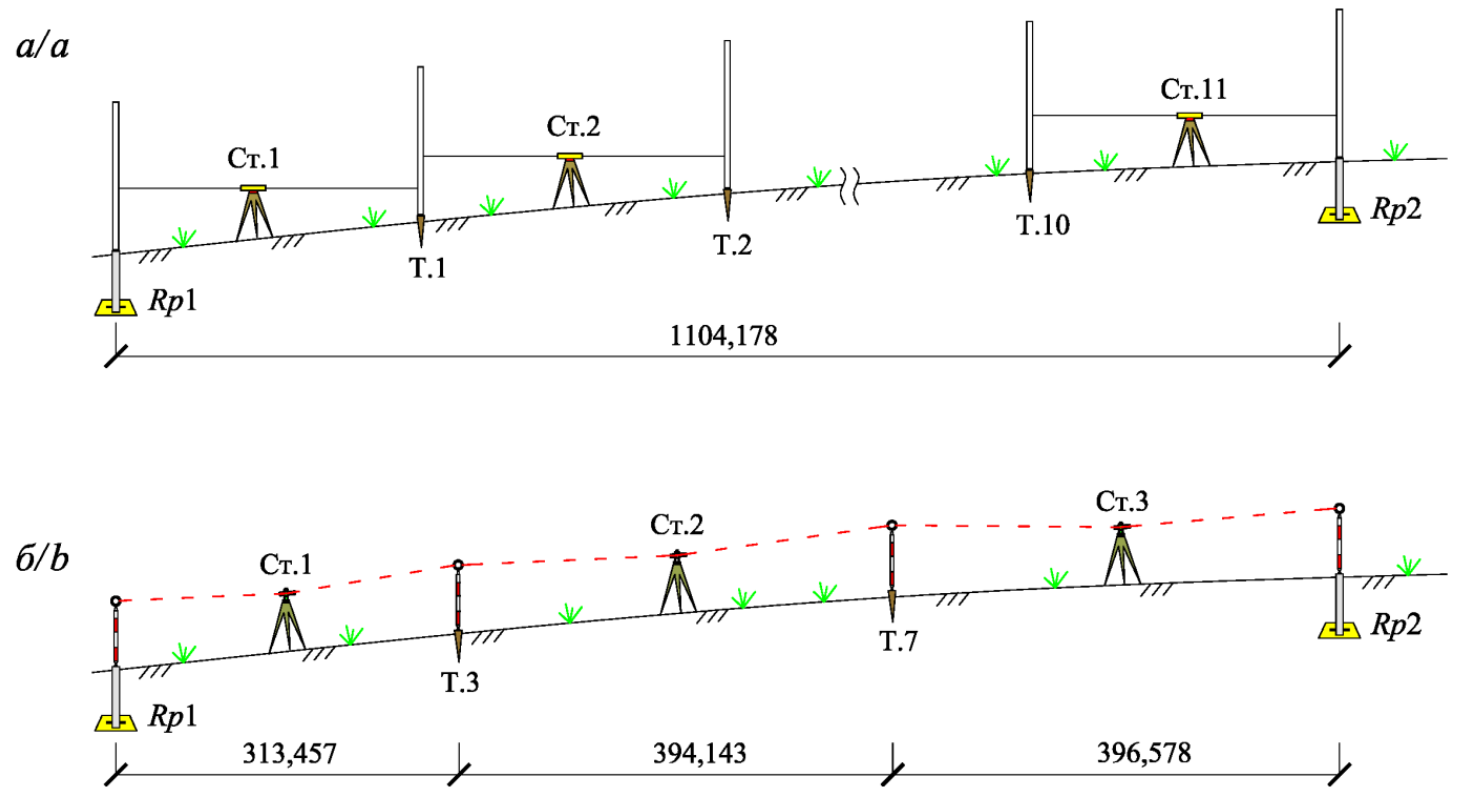

Рис. 7. Схема геометрического (а) и тригонометрического нивелирования из середины (б)

Fig. 7. Scheme of the geometric (a) and trigonometric leap-frog leveling (b)

Затем между исходными реперами был проложен ход тригонометрического нивелирования способом из середины (рис. 7, б), сначала по методике III класса (применялся тахеометр Leica TS-06 с паспортной точностью $m_{\alpha}=2^{\prime \prime}$ ), а на следующий день - по методике IV класса, тахеометром Leica TS-02 $\left(m_{\alpha}=5^{\prime \prime}\right)$. В обоих случаях измерения выполнялись в условиях переменной облачности, при температуре воздуха $+20 \ldots 25^{\circ} \mathrm{C}$.

В качестве визирных целей использовались стандартные круглые отражатели, закрепленные на вехах. Высота обоих отражателей была одинаковой $(l \approx 1,6$ м) Превышение на станции измерялось двумя приемами при двух положениях вертикального круга. В обработку брались средние значения из двух приемов, если их разности не превышали 5 и 7 мм, соответственно для III и IV классов (табл. 8).
Из табл. 8 следует, что уклонения превышений из тригонометрического нивелирования от эталонных значений находятся в пределах допусков, которые вычислялись соответственно для нивелирования III и IV классов по формулам

$$
\Delta_{\text {доп }}^{\mathrm{III}}=10 \mathrm{MM} \sqrt{L_{\text {км }}}, \quad \Delta_{\text {доп }}^{\mathrm{IV}}=20 \mathrm{MM} \sqrt{L_{\text {км }}} .
$$

Невязка хода тригонометрического нивелирования III класса, проложенного в двух направлениях, составила $f_{\mathrm{III}}=-2,86 \mathrm{Mм}$, а хода IV класса $-f_{\mathrm{IV}}=+5,74$ мм, что также не превосходит допустимых значений.

На основании проведенного эксперимента можно сделать вывод о реальной возможности выполнения нивелирования III и IV классов электронными тахеометрами. 
Таблица 8. Результаты сравнения геометрического и тригонометрического нивелирования

Table 8. Results of comparison of geometric and trigonometric leveling

\begin{tabular}{|c|c|c|c|c|c|c|c|}
\hline \multirow{3}{*}{$\begin{array}{l}\text { Точки высотного обоснования } \\
\text { Points of vertical control }\end{array}$} & \multicolumn{3}{|c|}{$\begin{array}{c}\text { Превышение, мм, определенное из нивелирования } \\
\text { Elevation, mm, determined from leveling }\end{array}$} & \multicolumn{4}{|c|}{$\begin{array}{c}\text { Уклонения } \Delta \text { от эталонного значения, мм } \\
\text { Deviations } \Delta \text { from standard value, } \mathrm{mm}\end{array}$} \\
\hline & \multirow{2}{*}{$\begin{array}{c}\text { геометрическое } \\
\text { (эталонное) } \\
\text { geometric } \\
\text { (standard) }\end{array}$} & \multicolumn{2}{|c|}{$\begin{array}{l}\text { тригонометрическое } \\
\text { trigonometric }\end{array}$} & \multicolumn{2}{|c|}{$\begin{array}{c}\text { фактические } \\
\text { actual }\end{array}$} & \multicolumn{2}{|c|}{$\begin{array}{c}\text { допустимые } \\
\text { admissible }\end{array}$} \\
\hline & & $\begin{array}{l}\text { III класс* } \\
\text { III class } \\
\end{array}$ & $\begin{array}{l}\text { IV класс } \\
\text { IV class }\end{array}$ & $\begin{array}{l}\text { III класс } \\
\text { III class }\end{array}$ & $\begin{array}{l}\text { IV класс } \\
\text { IV class }\end{array}$ & $\begin{array}{l}\text { III класс } \\
\text { III class }\end{array}$ & $\begin{array}{l}\text { IV класс } \\
\text { IV class }\end{array}$ \\
\hline$R p 1-\mathrm{T} .3$ & $+387,32$ & $+387,9$ & $+391,8$ & $+0,58$ & $+4,48$ & $\pm 5,6$ & $\pm 11,2$ \\
\hline T.3-T.7 & $+715,11$ & $+713,8$ & $+711,7$ & $-1,31$ & $-3,41$ & $\pm 6,3$ & $\pm 12,6$ \\
\hline T.7-Rp2 & $+349,43$ & $+347,3$ & $+354,1$ & $-2,13$ & $+4,67$ & $\pm 6,3$ & $\pm 12,6$ \\
\hline$\Sigma$ & $+1451,86$ & $+1449,0$ & 1457,6 & $-2,86$ & $+5,74$ & $\pm 10,5$ & $\pm 21,0$ \\
\hline
\end{tabular}

*Значения превышений получены как среднее из прямого и обратного ходов.

*Values are determined as average from forward and backward level run.

В настоящее время высокоточное нивелирование целесообразно применять для наполнения корпоративных ГИС данными о высотном положении объектов МТ и использовать в комплексе с данными космических съемочных систем с высоким и средним пространственным разрешением, а также материалами съемки с БПЛА и ВЛС [16, 23].

\section{Выводы}

1. Базы пространственных данных об объектах МТ должны поддерживаться в актуальном состоянии. Для решения задачи инвентаризации и контроля фактического положения объектов МТ целесообразно использовать данные космической съемки сверхвысокого разрешения $(<1 \mathrm{M})$, а также материалы АФС и съемки с БПЛА, обеспечивающие необходимую точность и детализацию для корректного распознавания объектов и уточнения их пространственного положения, состояния объектов и окружающей обстановки.

2. Состав данных ДЗ3 для обеспечения геопространственных данных для трехмерного моделирования земной поверхности и инфраструктуры трубопроводных систем предполагает три основных массива данных, которые условно можно разбить на три уровня:

- I-й уровень. Обзорное покрытие космической съемкой среднего разрешения (5-30 м) терри-

\section{СПИСОК ЛИТЕРАТУРЫ}

1. Возможности визуального дешифрирования магистральных трубопроводов и объектов инфраструктуры по спутниковым изображениям высокого и сверхвысокого пространственного разрешения / Д.В. Долгополов, Д.В. Никонов, А.В. Полуянова, В.А. Мелкий // Вестник СГУГиТ. - 2019. - Т. 24 - № 3. - С. 65-81.

2. Аэрофотосъемка // Сайт ZALA AERO GRUP. URL: https://zala-aero.com/services/aerial-photography/ (дата обращения 15.03.2020).

3. Zhongkun Zhang. Study on urban underground streets in modern cities // American Journal of Civil Engineering. - 2018. - V. 6. № 2. - P. 60-67. DOI: 10.11648/j.ajce.20180602.12

4. Thompson R., Oosterom P., Soon K.H. Mixed 2D and 3D Survey plans with topological encoding // The 5th International FIG 3D Cadastre Workshop. - Athens, Greece, 2016. URL: http://www.gdmc.nl/3DCadastres/literature/3Dcad_2016_17.pdf (дата обращения 15.03.2020).

5. Karatas K. Land recording of objects subject to 3D cadastre in Turkey // The World Cadastre Summit - Congress \& Exhibition. - тории РФ из открытых источников (например, Landsat и Sentinel);

- II-й уровень. Базовое покрытие космической съемкой высокого разрешения (1-5 м) линейной части МТ и прилегающих территорий с объектами в соответствии с потребностями пользователей;

- III-й уровень. Детальные материалы дистанционного зондирования сверхвысокого разрешения $(<1 \mathrm{M})$, полученные с носителей различного типа (космическая съемка, аэросъемка, съемка с БПЛА), выполняемые по запросам подразделений компаний, осуществляющих эксплуатацию и мониторинг трубопроводных систем.

3. При создании высотного геодезического обоснования с точностью III и IV классов вместо трудоемкого геометрического нивелирования предлагается применять метод тригонометрического нивелирования с использованием высокоточных электронных тахеометров. Наибольший эффект от применения предлагаемой методики тригонометрического нивелирования ожидается на заболоченной, пересеченной или холмистой местности, что особенно актуально для районов Крайнего Севера. Благодаря увеличению длины визирного луча до 200-300 м обеспечивается повышение производительности работ до двух раз.

Istanbul, Turkey, 2015. URL: http://www.gdmc.nl/3DCadastres/ literature/3Dcad_2015_10.pdf (дата обращения 15.03.2020).

6. Construction geometric model and topology for $3 \mathrm{~d}$ cadastre - case study in Taizhou, Jiangsu / Y. Ding, C. Wu, N. Jiang, B. Ma, X. Zhou / FIG Working Week 2016. URL: http://www.gdmc.nl/ 3DCadastres/ literature/3Dcad_2016_06.pdf (дата обращения 15.03.2020).

7. Ribeiro A., Almeida J., Ellul C. Exploring city engine as a visualisation tool for 3D cadaster // 4th International Workshop on FIG 3D Cadastres. - Dubai, United Arab Emirates, 2014. URL: http://www.gdmc.nl/3DCadastres/literature/3Dcad_2014_25.pdf (дата обращения 15.03.2020).

8. Разработка технологической схемы геодезических работ при постановке на кадастровый учет подземных парковочных мест / Г.А. Уставич, П.П. Сальникова, В.Г. Сальников, Е.Л. Соболева // Вестник СГУГиТ. - 2018. - Т. 23 (1). - С. 54-64.

9. Технологические аспекты построения 3D-модели инженерных сооружений в городах арктического региона РФ / Е.И. Аврунев, А.В. Чернов, А.В. Дубровский, А.В. Комиссаров, Е.Ю. Пасечник // Известия Томского политехнического университета. - 2018. - Т. 329. - № 7. - С. 131-137. 
10. Mobile precise trigonometric levelling system based on land vehicle: an alternative method for precise leveling / J. Zou, Y. Zhu, Y. Xu, Q. Li, L. Meng, H. Li// Survey Review. - 2017. V. 49. - Iss. 355. - P. 249-258.

11. Ceylan A., Baykal O. Precise height determination using simultaneous-reciprocal trigonometric leveling // Survey Review. - 2008. - V. 40 (308). - P. 195-205. DOI: $10.1179 / 003962608 X 290997$.

12. Kovačič B., Kamnik R. Accuracy of trigonometric heighting and monitoring the vertical displacements // Engineering modelling. 2007. - № 20. - P. 77-84.

13. Nestorovic Z., Delcev S. Comparison of height differences obtained by trigonometric and spirit leveling method // Geonauka. - 2014. - V. 2. - № 4. - P. 30-37.

14. Никонов А. В. Исследование точности измерения расстояний электронными тахеометрами в безотражательном режиме // Вестник СГУГиТ. - 2015. - Вып. 1 (29). - С. 43-53.

15. Křemen T., Štroner M., Třasák P. Determination of pavement elevations by the 3D scanning system and its verification // INGEO 2014 - 6th International Conference on Engineering Surveying. - Prague, Czech republic, April 3-4, 2014. - P. 69-74.

16. Опритова О.А. Исследование возможностей применения беспилотных авиационных систем для моделирования объектов недвижимости // Вестник СГУГиТ. - 2018. - Т 23 (3). C. $248-258$.

17. Rueger J.M. EDM-height traversing refraction correction and experiences // Trans Tasman Surveyor. - June, 1995. - V. 1. № 1. - P. 48-56.
18. Rueger J.M., Brunner F.K. Practical results of EDM-height traversing // The Australian Surveyor. - June, 1981. - V. 30. № 6. - P. 363-372.

19. Никонов А.В. Совершенствование методики тригонометрического нивелирования короткими лучами: дис. ... канд. техн. наук. - Новосибирск, 2015. - 261 с.

20. Никонов А.В. Исследование влияния вертикальной рефракции на результаты тригонометрического нивелирования короткими лучами способом из середины // Изв. вузов. Геодезия и аэрофотосъемка. - 2014. - № 1. - С. 28-34.

21. Monitoring of the refraction coefficient of the lower atmosphere using a controlled set-up of simultaneous reciprocal vertical angle measurements / C. Hirt, S. Guillaume, A. Wisbar, B. Bürki, H. Sternberg // Journal of Geophysical Research (JGR). - 2010. V. 115. - D21102. DOI: 10.1029/2010JD014067.

22. Zienkiewicz M.H., Baryła R. Determination of vertical indicators of ground deformation in the Old and Main City of Gdansk area by applying unconventional method of robust estimation // Acta Geodyn. Geomater. - 2015. - № 3 (179). - P. 249-257.

23. Применение воздушного лазерного сканирования для геотехнического мониторинга объектов магистрального трубопровода / Е.М. Макарычева, Э.Р. Ибрагимова, Т.И. Кузнецова, К.Ю. Шуршина // Наука и технологии трубопроводного транспорта нефти и нефтепродуктов. - 2019. - Т. 9. - № 1. C. 21-31. DOI: $10.28999 / 2541-9595-2019-9-1-21-31$

Поступила: 26.03.2020 2.

\section{Информация об авторах}

Аврунев Е.И., кандидат технических наук, доцент кафедры кадастра и территориального планирования Сибирского государственного университета геосистем и технологий.

Уставич Г.А., доктор технических наук, профессор кафедры инженерной геодезии и маркшейдерского дела, Сибирского государственного университета геосистем и технологий.

Грекова A.O., магистр института кадастра и природопользования Сибирского государственного университета геосистем и технологий.

Никонов А.В., кандидат технических наук, ведущий инженер АО «Сибтехэнерго»; доцент кафедры инженерной геодезии и маркшейдерского дела Сибирского государственного университета геосистем и технологий.

Meлкий B.A., доктор технических наук, ведущий научный сотрудник лаборатории вулканологии и вулканоопасности Института морской геологии и геофизики ДВО РАН.

Долгополов Д.В., кандидат технических наук, ведущий научный сотрудник АО «СпейсИнфо Геоматикс». 
UDC528.8/.37/.38

\section{TECHNOLOGICAL SOLUTIONS IN SPHERE OF GEOSPATIAL INFORMATION ON LONG DISTANCE PIPELINES AND OBJECTS OF THEIR INFRASTRUCTURE}

\section{Evgeny I. Avrunev 1 , avrynev_ei@ngs.ru}

\author{
Georgy A. Ustavich ${ }^{1}$, \\ ystavich@mail.ru
}

\section{Antonina 0. Grekova ${ }^{1}$, antoninaop@mail.ru}

\author{
Anton V. Nikonov1,2, \\ sibte@bk.ru
}

\author{
Vyacheslav A. Melkiy ${ }^{3}$ \\ vamelkiy@mail.ru
}

Daniil V. Dolgopolov4, daniil.dolgopolov@gmail.com

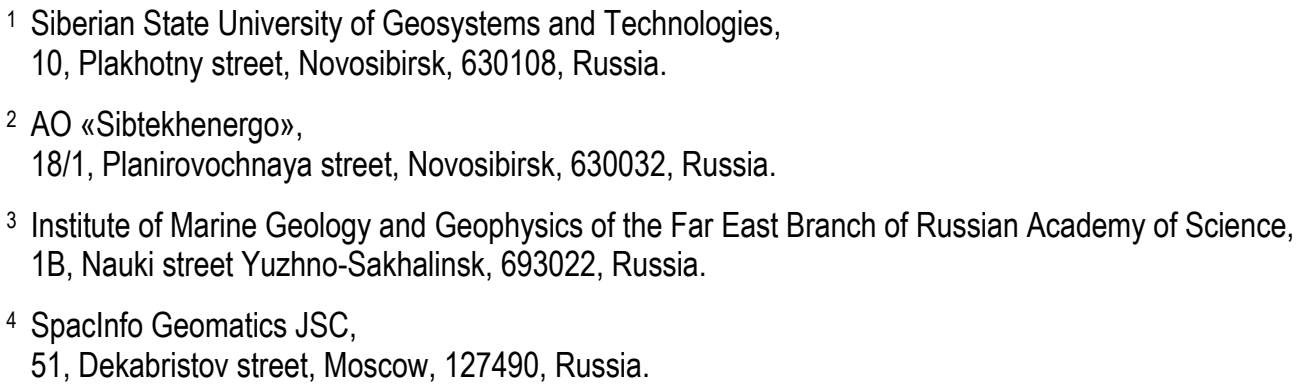

Relevance of the research is caused by the fact that currently in the Russian Federation, for servicing mining and development of minerals, it is necessary to perform survey and geodetic works, by the results of which arrays of geospatial data are formed. They are the basis for the design and arrangement of oil and gas fields and are the source information for three-dimensional modeling of the earth's surface, geological structures of deposits and infrastructure of pipeline systems. Besides, geospatial data is necessary for preparation of documents for state cadastral registration and registration of land use rights. Based on this, the article sets scientific and technical task: analyze the possibilities of using various methods of providing geospatial information GIS pipeline systems with data for 3D modeling of the studied fields and pipelines. Corporate GIS is filled with data on the state of pipeline transport, using information from space survey systems with high and medium spatial resolution, as well as shooting materials from unmanned aerial vehicles and aerial laser scanning. Monitoring of pipeline systems is carried out as well using ground-based geodetic survey data. One of the most common types of modern geodetic equipment is high-precision and accurate electronic total stations that allow obtaining geospatial information on terrain and anthropogenic objects both in terms of plan and height. The height component is determined as result of performing trigonometric leveling. The use of the trigonometric leveling method is especially relevant when working in rough terrain and adverse conditions, in particular in regions of the Extreme North.

The aim of the research is to analyze the possibilities of using various methods of providing geospatial information for GIS pipeline systems: satellite surveys with different spatial resolutions, unmanned aerial vehicles and aerial laser scanning data, trigonometric leveling of classes III and IV with the use of high-precision electronic total stations in the formation of geospatial data arrays of main pipeline monitoring systems.

Methods: decoding of aerospace images, experimental research in field with space survey materials, unmanned aerial vehicles and aerial laser scanning data, use of high-precision levels and electronic total stations for trigonometric leveling, geoinformation technologies

Results. Quality of geospatial information for GIS monitoring of pipeline systems was evaluated. The work shown that the data from remote sensing of the Earth with different spatial resolutions, unmanned aerial vehicles and aerial laser scanning allow forming arrays of geospatial data necessary for services operating pipeline systems, providing planned linking with sufficient accuracy; when creating highaltitude geodetic justification, when accuracy needs be increased to class III and IV, instead of time-consuming geometric leveling, it is advisable to supplement arrays with information, obtained by trigonometric leveling using high-precision electronic tacheometers.

\section{Key words:}

Geospatial data, decoding of aerospace images, geoinformation technologies, unmanned aerial vehicles, aerial laser scanning, three-dimensional model of the earth's surface, trigonometric leveling, high-precision electronic tacheometer.

\section{REFERENCES}

1. Dolgopolov D.V., Nikonov D.V., Poluyanova A.V., Melkiy V.A. Possibilities of visual interpretation of trunk pipelines and infrastructure facilities using satellite images of high and ultrahigh spatial resolution. Vestnik of the Siberian State University of Geosystems and Technologies (SSUGT), 2019, vol. 24, no. 3, pp. 6581. In Rus. DOI: 10.33764/2411-1759-2019-24-3-65-81

2. Aerial photography. Website ZALA AERO GRUP. Available at: https://zala-aero.com/services/aerial-photography/ (accessed 15 March 2020).

3. Zhongkun Zhang. Study on Urban Underground Streets in Modern Cities. American Journal of Civil Engineering, 2018, vol. 6, no. 2, pp. 60-67. DOI: $10.11648 /$ j.ajce.20180602.12
4. Thompson R., Oosterom P., Soon K.H. Mixed 2D and 3D survey plans with topological encoding. The $5^{\text {th }}$ International FIG $3 D$ Cadastre Workshop. Athens, Greece, 18-20 October 2016. Available http://www.gdmc.nl/3DCadastres/literature/3Dcad_2016_17.pdf (accessed 15 March 2020).

5. Karatas K. Land recording of objects subject to 3D cadastre in Turkey. The World Cadastre Summit - Congress \& Exhibition. Istanbul, Turkey, 20-25 April 2015. Available at: http://www.gdmc.nl/3DCadastres/literature/3Dcad_2015_10.pdf (accessed 15 March 2020).

6. Ding Y., Wu C., Jiang N., Ma B., Zhou X. Construction geometric model and topology for $3 \mathrm{~d}$ cadastre - case study in Taizhou, 
Jiangsu. FIG Working Week 2016. Recovery from Disaster. Christchurch, New Zealand, May 2-6, 2016. Available at: http://www.gdmc.nl/3DCadastres/literature/3Dcad_2016_06.pdf (accessed 15 March 2020).

7. Ribeiro A., Almeida J., Ellul C. Exploring city engine as a visualisation tool for 3D cadastre. $4^{\text {th }}$ International Workshop on FIG 3D Cadastres. Dubai, United Arab Emirates, 9-11 November 2014. Available at: http://www.gdmc.nl/3DCadastres/literature/ 3Dcad_2014_25.pdf (accessed 15 March 2020).

8. Ustavich G.A., Sal'nikova P.P., Sal'nikov V.G., Soboleva E.L. Development of the technological scheme of surveying works for cadastral registration of underground parkings. Vestnik of the Siberian State University of Geosystems and Technologies (SSUGT), 2018, vol. 23 (1), pp. 54-64. In Rus.

9. Avrunev E.I., Chernov A.V., Dubrovsky A.V., Komissarov A.V., Pasechnik E.Yu. Technological aspects of constructing 3D-model of engineering structures in the cities of the RF Arctic Region. Bulletin of the Tomsk Polytechnic University. Geo Assets Engineering, 2018, vol. 329, no. 7, pp. 131-137. In Rus.

10. Zou J., Zhu Y., Xu Y., Li Q., Meng L., LiH. Mobile precise trigonometric levelling system based on land vehicle: an alternative method for precise leveling. Survey Review, 2017 $\begin{array}{llll}\text { vol. } 49, & \text { Iss. } 355, & \text { pp. 249-258. }\end{array}$ doi.org/10.1080/00396265.2016.1163877

11. Ceylan A., Baykal O. Precise height determination using simultaneous-reciprocal trigonometric leveling. Survey Review, 2008, vol. 40 (308), pp. 195-205 DOI: 10.1179/003962608X290997.

12. Kovačič B., Kamnik R. Accuracy of trigonometric heighting and monitoring the vertical displacements. Engineering modeling, 2007, no. 20, 1-4, pp. 77-84.

13. Nestorovič Z., Delčev S. Comparison of height differences obtained by trigonometric and spirit leveling method. Geonauka, 2014, vol. 2, no. 4, pp. 30-37. DOI: 10.14438/gn.2015.01

14. Nikonov A.V. Study of reflectionless total stations distance measurement accuracy. Vestnik of the Siberian State University of Geosystems and Technologies (SSUGT), 2015, vol. 1 (29), pp. 4353. In Rus.
15. Křemen T., Štroner M., Třasák P. Determination of pavement elevations by the $3 \mathrm{D}$ scanning system and its verification. INGEO 2014 - $6^{\text {th }}$ International Conference on Engineering Surveying. Prague, Czech Republic, April 3-4, 2014. pp. 69-74. DOI: 10.14311/gi.12.9.

16. Opritova O.A. Studies of possibilities on using unmanned aircraft systems for real estate modeling. Vestnik of the Siberian State University of Geosystems and Technologies (SSUGT), 2018, vol. 23 (3), pp. 248-258. In Rus.

17. Rueger J.M. EDM-height traversing refraction correction and experiences. Trans Tasman Surveyor, June 1995, vol. 1, no. 1, pp. $48-56$.

18. Rueger J.M., Brunner F.K. Practical results of EDM-height traversing. The Australian Surveyor, June 1981, vol. 30, no.6, pp. 363-372.

19. Nikonov A. V. Sovershenstvovanie metodiki trigonometricheskogo nivelirovaniya korotkimi luchami. Dis. Kand. nauk [Improving the technique of trigonometric leveling with short beams. Cand. Diss.]. Novosibirsk, 2015. 261 p.

20. Nikonov A.V. Research of vertical refraction influence upon trigonometric leveling by short beams from the middle. Scientific journal Izvestia vuzov "Geodesy and aerophotosurveying», 2014, no. 1, pp. 28-34. In Rus.

21. Hirt C., Guillaume S., Wisbar A., Bürki B., Sternberg H. Monitoring of the refraction coefficient of the lower atmosphere using a controlled set-up of simultaneous reciprocal vertical angle measurements. Journal of Geophysical Research (JGR), 2010, vol. 115, D21102. DOI: 10.1029/2010JD014067.

22. Zienkiewicz M.H., Baryła R. Determination of vertical indicators of ground deformation in the Old and Main City of Gdansk area by applying unconventional method of robust estimation. Acta Geodyn. Geomater, 2015, no. 3 (179), pp. 249-257.

23. Makarycheva E.M., Ibragimov E.R., Kuznetsov T.I., Shurshin K.Y. Using the airborne laser scanning method for geotechnical monitoring of pipeline system facilities. Science \& Technologies [Oil and Oil Products Pipeline Transportation], 2019, no. 9 (1), pp. 21-31. In Rus. DOI: 10.28999/2541-9595-2019-9-1-21-31

Received: 26 March 2020.

\section{Information about the authors}

Evgeny I. Avrunev, Cand. Sc., associate professor, Siberian State University of Geosystems and Technologies.

Georgy A. Ustavich, Dr. Sc., professor, Siberian State University of Geosystems and Technologies.

Antonina O. Grekova, master of science, Siberian State University of Geosystems and Technologies.

Anton V. Nikonov, Cand. Sc., chief engineer, AO «Sibtekhenergo»; Siberian State University of Geosystems and Technologies.

Vyacheslav A. Melkiy, Dr. Sc., leading researcher, Institute of Marine Geology and Geophysics of the Far East Branch of Russian Academy of Science

Daniil V. Dolgopolov, Cand. Sc., leading researcher, SpacInfo Geomatics JSC. 